\title{
Neuromuscular Adaptations in Isokinetic, Isotonic, and Agility Training Programs*
}

\author{
Edward M. Wojtys, † MD, Laura J. Huston, MS, Paul D. Taylor, and Steven D. Bastian \\ From MedSport, the Section of Orthopaedic Surgery, University of Michigan, \\ Ann Arbor, Michigan
}

\begin{abstract}
Weight training is an integral part of most athletic conditioning programs; yet, the effect of these programs on neuromuscular function remains unclear. To examine the neuromuscular effects of training and conditioning at the knee joint, 32 volunteers (16 men and 16 women; average age, 25.4 years) were placed into one of four groups: isokinetic, isotonic, agility, or control. Each group trained 3 days per week for 6 weeks. The knee function of all participants was evaluated just before and after the 6-week training period. The agilitytrained group significantly improved the spinal reflex times of the lateral and medial quadriceps muscles in response to anterior tibial translation. The cortical response time of the agility group also significantly improved in the gastrocnemius, medial hamstring, and the lateral quadriceps muscles. Interestingly, the cortical response time of the medial hamstring and the medial quadriceps muscles in the isokinetic group slowed significantly, by 39.1 and $32.4 \mathrm{msec}$, respectively, after 6 weeks of training. Isotonic and isokinetic strength training of the lower extremities do not appear to improve muscle reaction time to anterior tibial translation, whereas agility exercises potentially improve this parameter.
\end{abstract}

Weight training and physical conditioning have become year-round requirements for sports, such as football and basketball, at the upper levels of competition. The physiologic effects of these progressive resistance exercise programs ${ }^{10}$ have been investigated extensively, including the hormonal ${ }^{13}$ and electromyographic ${ }^{7,12}$ changes that result from training. These programs are designed to im-

*Presented at the 20th annual meeting of the AOSSM, Palm Desert, California, June 1994.

†Address correspondence and reprint requests to Edward M. Wojtys, MD, MedSport, POB 363, Ann Arbor, MI 48106.

No author or related institution has received any financial benefit from research in this study. prove muscle strength, endurance, and skill while decreasing the chance of injury at susceptible joints such as the knee. ${ }^{8}$ Strength and endurance are important muscle performance criteria for athletic endeavors; however, the biomechanical mechanisms by which these features protect the extremities against injury remain unclear.

Traditionally, strength has been emphasized as the most important element in training and conditioning programs designed to protect the knee. Muscle strength is quantifiable, and it is comparable with other anthropometric data, such as body weight. Strength in the quadriceps, hamstring, and gastrocnemius muscles can protect a knee only when joint compression is achieved in a timely fashion, which allows knee joint surface congruency to become a stabilizing element by resisting rotation or shear forces. Therefore, muscle reaction times and the time needed to generate peak muscle torque appear to be important parameters to consider in injury prevention because they determine how rapidly dynamic control can be activated to stabilize the knee against deforming forces. ${ }^{5}$

The neuromuscular effects of athletic training in the ACL-deficient knee have been investigated and direct correlations were found among muscle reaction time, instability symptoms, and athletic performance. ${ }^{21}$ How crucial muscle reaction times are to normal extremities for joint stabilization and injury prevention remains unknown ${ }^{2,4}$; yet, the clinical importance of muscle reaction times seems obvious. ${ }^{3}$

The primary goal of our investigation was to determine the effects of various exercise regimens (isotonic, isokinetic, and agility) on the muscle reaction time and time to peak muscle torque of the muscles crossing the knee joint (i.e., the quadriceps, hamstring, and gastrocnemius muscles) that are capable of knee joint compression and may ultimately resist anterior tibial translation.

\section{MATERIALS AND METHODS}

\section{Epidemiology}

Thirty-two healthy volunteers (16 men and 16 women; average age, 25.4 years) were placed into one of four 
groups: isokinetic, isotonic, agility, or control. We thought that because of the required intense training, self-motivation was a critical element to the success of the study. Therefore, group selection was based on patient choice rather than by random assignment. With the exception of one member of the control group, all subjects were rightleg dominant (dominance was determined by the leg used to kick a ball). In addition, there were no significant differences in age or activity level between each training group and the control group. All three training groups exercised for 30 minutes three times per week for 6 weeks.

\section{Isokinetic Protocol}

The isokinetic protocol included knee flexion and extension and ankle plantar flexion and dorsiflexion exercises on an isokinetic dynamometer (Biodex Corp., Shirley, New York). Peak muscle torque (strength), work fatigue (endurance), and time to peak torque were recorded. All strength and endurance levels were standardized for the body weight of the volunteer (torque[in foot-pounds]/body weight[in pounds] $\times 100 \%$ ). The protocol consisted of 3 sets of 12 repetitions at $60 \mathrm{deg} / \mathrm{sec}$ for each exercise. This relatively slow speed was chosen because greater improvements in peak torque have been reported with training at $60 \mathrm{deg} / \mathrm{sec}$ than with faster isokinetic speeds. ${ }^{1,6}$

\section{Isotonic Protocol}

The isotonic protocol consisted of 3 exercises (using the maximum weight resistance tolerated for 3 sets of 12 repetitions): leg press (knee extension), hamstring muscle curls (knee flexion), and calf muscle raises (ankle plantar flexion). For the leg press, the subjects sat at the leg press machine with their knees at $90^{\circ}$ of flexion while pushing the weight to within $10^{\circ}$ of full knee extension. The workout of the gastrocnemius and soleus muscles was also done on the leg press machine. The knees were at full extension while the midfoot was placed on the foot plate, thus allowing full plantar flexion and dorsiflexion at the ankle. The hamstring muscle curls were performed with the subjects in the seated position and their knees flexed from $0^{\circ}$ to $90^{\circ}$. All subjects recorded their resistance improvements to monitor daily progress.

\section{Agility Training Protocol}

The agility training protocol consisted of five drills regularly used in ACL rehabilitation programs and had been used in a previous pilot study. These drills attempt to strengthen and coordinate the lower extremity muscle groups that stabilize the knee during physical activity. The drills were as follows: 1) slideboarding (5 sets of 25 crossings), 2) unilateral bounding "box hop" jumps (3 sets of 10 repetitions), 3) carrioca (i.e., foot crossover drills, 3 sets of 10 repetitions), 4) Figure-of- 8 runs ( 3 sets of 5 circuits in both directions), and 5) backward runs ( 3 sets of 10 repetitions at 15 feet). The agility group performed each exercise as quickly and as accurately as possible. Progress was measured by the time needed to complete each exercise.

\section{Control Group}

The control group participated only in activities of daily living and the recreational sports that they were involved in before the study. However, the control group refrained from any sports-related muscle training or conditioning during the 6-week training period.

\section{Testing Protocol}

All participants underwent arthrometer (KT-1000, MEDmetric Corp., San Diego, California) anterior tibial translation testing, isokinetic peak torque and endurance tests at 60 and $240 \mathrm{deg} / \mathrm{sec}$ for knee flexion and extension, 60 and $180 \mathrm{deg} / \mathrm{sec}$ for ankle plantar flexion, and an anterior tibial translation stress test at the knee with surface EMG monitoring. ${ }^{21}$

\section{Anterior Tibial Translation Stress Test}

This test was designed to measure anterior tibial translation in reference to the femur in response to an anteriorly directed 30-pound step force applied to the posterior aspect of the proximal tibia with the lower extremity muscles 1) relaxed and 2) responding to the displacement (Fig. 1). Surface electromyography was recorded at the following five muscle locations during force application: gastrocnemius, lateral hamstring, medial hamstring, lateral quadriceps, and medial quadriceps. Tibial displacement relative to the femur was quantified by two linear potentiometers: the first on the patella and the second on the tibial tuberosity.

During testing at $30^{\circ}$ of knee flexion, participants were seated on a support specifically designed to allow uninhibited hamstring muscle activity. The foot was strapped to a scale to monitor weightbearing status (maintained at 20 to 30 pounds) while the ankle was kept in $10^{\circ}$ to $15^{\circ}$ of dorsiflexion. Two bipolar surface electrodes (Hewlett Packard, Waltham, Massachusetts) were placed longitudinally over the midregion of each muscle group, $3 \mathrm{~cm}$ apart. The raw EMG signals were amplified at $100 \mu \mathrm{V} / \mathrm{V}$ and fed into a Zenith Data Systems 286 computer (Zenith Data Systems, St. Joseph, Michigan) for acquisition and analysis. The EMG recordings were sampled at a frequency rate of $1000 \mathrm{~Hz}$ during a 2.5-second time frame beginning 0.5 seconds before the onset of the anteriorly directed force.

Two types of muscle tests, relaxed and response, were performed on each subject. One test was performed with the lower extremity muscles at rest (i.e., relaxed) while the force was applied. If muscle activity was detected on EMG readings before force application, the test was discarded and repeated. The muscle response tests were performed by asking the participants to contract their lower extremity muscles as soon as they detected the anterior tibial displacement. Ten trials of each test were recorded 


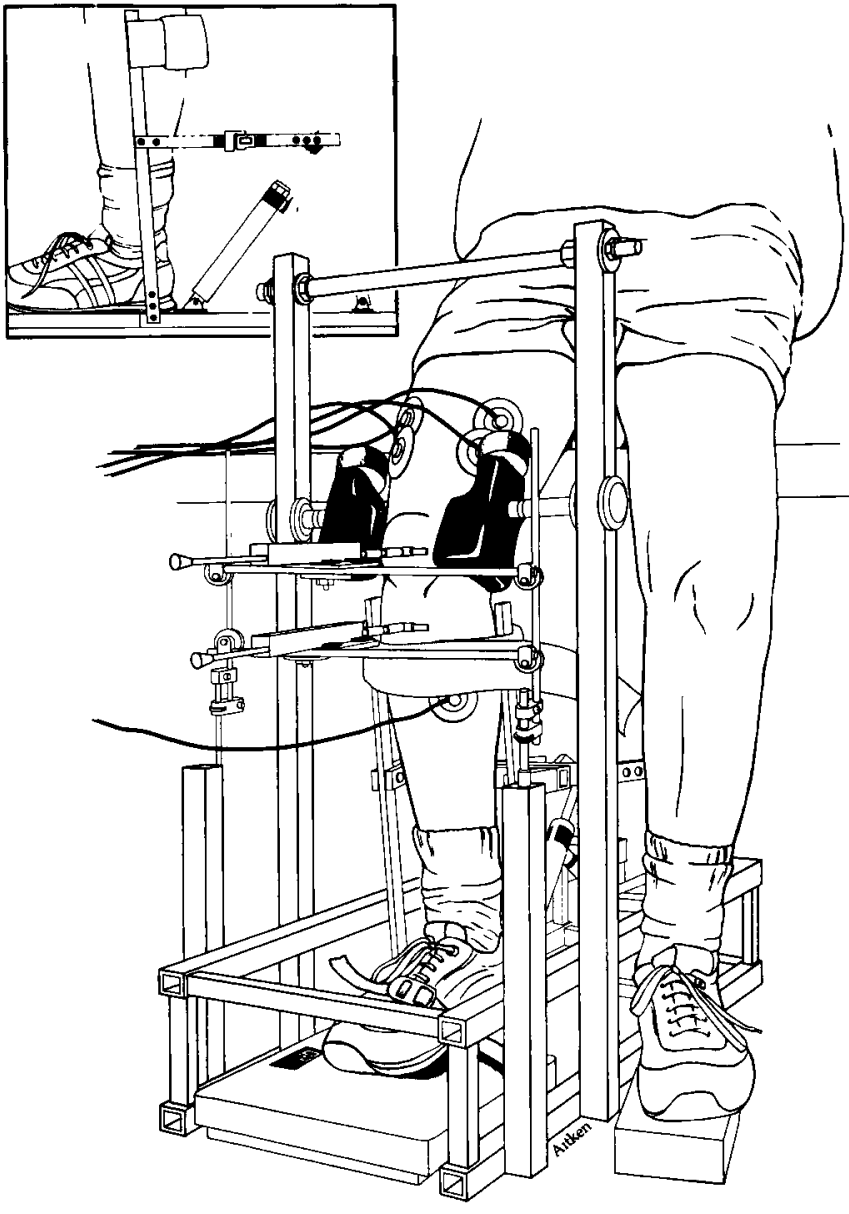

Figure 1. Knee testing apparatus measures anterior tibial translation in reference to the femur in response to an anteriorly directed 30 -pound step force applied to the posterior aspect of the leg.

and the results were averaged. To prevent anticipation of anterior force application, randomized time delays (range, 0.1 to 10 seconds) were used before force application. All visual and audio clues associated with the force application were eliminated. The reproducibility of this anterior tibial translation stress test was reported previously by Wojtys and Huston. ${ }^{21}$

\section{Data Analysis}

The muscle reaction times were calculated by measuring the time delay between the onset of the 30-pound stimulus and the beginning of muscle activity. Three regions of the EMG signal were analyzed as follows: 1) spinal reflex, 2) intermediate response, and 3) voluntary muscle activity. ${ }^{21}$ Each of these responses has specific time and shape characteristics that were used to identify three portions of the EMG recording. ${ }^{21}$ The spinal reflex is a monosynaptic response that resembles a tendon tap on a physical examination. This reflex occurred between 26 to $130 \mathrm{msec}$ after the initiation of the tibial displacement. The intermediate response appears to be a spinal reflex with interneuronal input from higher centers and resembles the late response seen during electric nerve stimulation. This response occurred between 110 and $216 \mathrm{msec}$ after the onset of the displacing force. The intermediate response is biphasic and routinely occurs just before voluntary activity. Voluntary muscle activity is always the largest in amplitude (two times that seen in the spinal reflex), biphasic, longest in duration, and it began between 156 and $431 \mathrm{msec}$ after the initiation of anterior tibial translation in this study.

\section{Statistics}

We performed a power analysis to determine the number of subjects needed in each group based on the results from a pilot study. One-way analysis of variance for repeated measures was used to identify differences, with Tukey's post hoc test used to determine significance between groups. An adjusted significance level of $P \leq 0.01$ was employed to account for the repeated measures procedures. Correlations were performed using the Pearson correlation matrix method with Bonferroni's adjusted probability.

\section{RESULTS}

\section{Knee Laxity}

No significant differences ( $P \leq 0.01$ ) were found between the control group and each of the three training groups in either KT-1000 arthrometer measurements or anterior tibial translation stress testing (muscles contracted) before or after 6 weeks of training. However, anterior tibial translation stress testing (muscles relaxed) in the isotonic group showed a statistically significant increase of 0.83 $\mathrm{mm}$ when compared with the control group after training.

\section{Strength}

All strength testing at 0 and 6 weeks (peak torque and endurance) was performed on an isokinetic dynamometer (Table 1). The isokinetic group was significantly stronger in knee extension and ankle plantar flexion peak torque after 6 weeks of training when compared with the control, agility, and isotonic groups. Knee extension strength increased $7.2 \%$ and ankle plantar flexion strength improved $18.9 \%$. No differences were found in the knee flexion peak torque after 6 weeks of training in the isokinetic group. Interestingly, no increase in isokinetic strength was found in the isotonic or the agility groups after the training

TABLE 1

Average Change in Strength after 6 Weeks of Training

\begin{tabular}{lcccc}
\hline \multicolumn{1}{c}{ Exercises } & Control & Agility & Isokinetic & Isotonic \\
\hline Knee extension & -2.2 & -0.7 & $7.2^{b}$ & -0.3 \\
Knee flexion & -0.6 & 0.9 & -0.3 & 1.5 \\
Ankle plantar flexion & -1.9 & 2.3 & $18.9^{b}$ & 3.8 \\
\hline
\end{tabular}

${ }^{a}$ Percentage of strength equals peak torque (in foot-pounds) divided by body weight (in pounds) times 100 .

${ }^{b}$ Significant compared with the control group at $P<0.01$ level. 
program. All strength values were normalized with respect to body weight to standardize results.

\section{Endurance}

There were no significant differences in the endurance rates of knee flexion, knee extension, or ankle plantar flexion in any of the groups either before or after training.

\section{Muscle Reaction Time}

Spinal Reflex. In the agility-trained group, the participants generated a spinal level reflex in response to anterior tibial translation in the medial and lateral quadriceps muscles an average of 13.8 and $15.4 \mathrm{msec}$ earlier than the other groups (Table 2). No other changes at the spinal cord level were significant.

Intermediate Response. The intermediate response of the medial hamstring muscles of the agility-trained group significantly improved by $18.1 \mathrm{msec}$ after training $(P \leq$ 0.01 ). No other statistically significant changes were found in other muscle groups of any training group (Table 2).

Voluntary Response. Three of the five muscles tested in the agility group demonstrated significant reductions in voluntary response times: gastrocnemius muscle (15.7 msec), medial hamstring muscle $(34.4 \mathrm{msec})$, and lateral quadriceps muscle (27.9 msec) (Table 2). The lateral hamstring and medial quadriceps muscles also improved after the 6-week program; however, this change was not statistically significant.

The response times in the isokinetic group were slower in all five muscle groups, with the medial hamstring (39.1

TABLE 2

Average Change in Muscle Reaction Time after 6 Weeks of Training (in milliseconds)

\begin{tabular}{lrrrr}
\hline & \multicolumn{4}{c}{ Groups } \\
\cline { 2 - 5 } Category $^{a}$ & Control & Agility & Isokinetic & Isotonic \\
\hline Spinal reflex & & & & \\
G & 7.0 & -5.8 & 6.6 & 8.9 \\
LH & -3.3 & -3.5 & -6.3 & -1.9 \\
MH & -5.7 & -2.3 & -1.3 & -7.5 \\
LQ & 8.9 & $-15.4^{b}$ & 9.3 & 6.2 \\
MQ & 16.8 & $-13.8^{b}$ & & 2.4 \\
Intermediate & & & & \\
response & & & & \\
G & -5.8 & -2.8 & 0.7 & -9.2 \\
LH & -4.2 & -5.7 & 0.3 & -4.0 \\
MH & 0.3 & $-18.1^{b}$ & -4.4 & -2.8 \\
LQ & -3.8 & -12.3 & 1.6 & -6.4 \\
MQ & -5.5 & -12.5 & 7.8 & -8.6 \\
Voluntary & & & & \\
reponse & & & & \\
G & 11.8 & $-15.7^{b}$ & 15.1 & 5.1 \\
LH & -4.1 & -20.0 & 21.7 & 0.5 \\
MH & -4.5 & $-34.4^{b}$ & $39.1^{b}$ & 2.5 \\
LQ & 0.8 & $-27.9^{b}$ & 0.4 & 8.3 \\
MQ & 5.7 & -9.7 & $32.4^{b}$ & 10.9 \\
\hline
\end{tabular}

${ }^{a} \mathrm{G}$, gastrocnemius; $\mathrm{LH}$, lateral hamstring; $\mathrm{MH}$, medial hamstring; LQ, lateral quadriceps; MQ, medial quadriceps.

${ }^{b}$ Significant compared with the control group at $P<0.01$ level. msec) and medial quadriceps (32.4 msec) muscles showing significant delays. The isotonic group's responses showed insignificant change after 6 weeks of training.

\section{Time to Peak Torque}

No statistically significant differences were found in time to peak muscle torque within or among groups after 6 weeks of training for knee extension, flexion, or ankle plantar flexion at $60 \mathrm{deg} / \mathrm{sec}$, but several trends were seen (Fig. 2). All training groups improved the time to peak muscle torque in knee extension, with the agility group showing the largest improvement ( $39 \mathrm{msec}$ ). The isotonic group showed the smallest change, with an average improvement of $9 \mathrm{msec}$. Interestingly, the control group improved an average of $11 \mathrm{msec}$.

The time to peak muscle torque for knee flexion in the agility group improved by the greatest margin $(38 \mathrm{msec})$. The times from the isotonic group were slower (31 msec).

The time to peak muscle torque for ankle plantar flexion in the isokinetic group improved by an average of $15 \mathrm{msec}$. The other two training groups were slower after 6 weeks of training (agility, $7 \mathrm{msec}$; isotonic, $6 \mathrm{msec}$ ).

The isokinetic group was the only training group to decrease the time to peak muscle torque in the quadriceps, hamstring, and gastrocnemius muscles. The time to peak muscle torque in ankle plantar flexion of the agilitytrained group significantly improved by $74 \mathrm{msec}$ at 180 $\mathrm{deg} / \mathrm{sec}$. The time to peak muscle torque in knee extension and flexion showed no change in any training group.

\section{Correlations}

The spinal, intermediate, and voluntary response times were compared with the knee extension, knee flexion, and ankle plantar flexion peak torque/body weight results. No strong correlations were found between strength and muscle reaction time using the Pearson correlation matrix method of comparison.

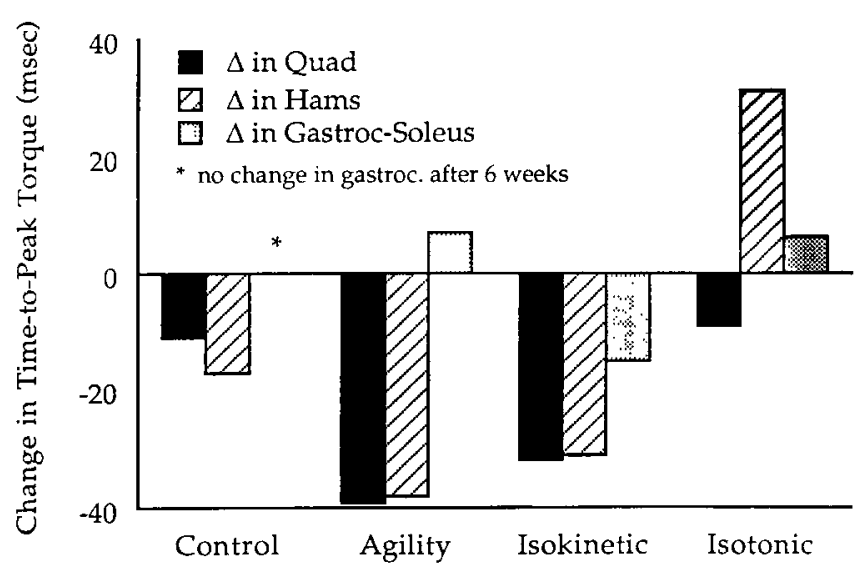

Figure 2. The average change in time to peak muscle torque in the quadriceps, hamstring, and gastrocnemius muscles between 0 and 6 weeks. 
Injuries

Unfortunately, an alarming number of participants in the isokinetic group developed knee pain during training. Three of the eight subjects in the isokinetic group developed patellar tendinitis and had to be removed from the study because of unacceptable levels of pain during the workouts. One subject in the agility group was removed from the study after 2 weeks because of a lateral ankle sprain that occurred while performing the carrioca drills.

\section{DISCUSSION}

The goal of our investigation was to determine which exercise regimen could improve the muscle reaction times of the quadriceps, hamstring, and gastrocnemius muscles needed to stabilize the knee joint against anterior tibial translation by joint compression and to see which exercise programs improve the time needed to reach peak muscle torque in muscles that cross the knee joint. Previous investigations of the effects of training on muscle reaction time, which did not involve anterior tibial translation, have produced mixed results. ${ }^{9,11}$ Ihara and Nakayama ${ }^{14}$ reported no improvement in muscle reaction time in knee muscles with isometric training, and Francis and Tiptron ${ }^{9}$ documented a $5 \%$ improvement in the quadriceps muscle reflex time with 6 weeks of isotonic training. The testing performed by Francis and Tiptron, however, used a tendon tap test rather than tibial translation, making data comparison with this study difficult.

All three training regimens in this study emphasized the quadriceps, hamstring, and gastrocnemius muscles in regular exercise sessions. Surprisingly, only the agilitytrained group had significant improvements in muscle reaction times at the spinal cord, intermediate, and voluntary muscle response levels. The voluntary muscle reaction time of all five muscles in the agility group improved; statistically significant changes resulted in three muscle groups. These improvements in muscle reaction time may initially appear minuscule, but they approach the length of time reported by Yasuda et al. ${ }^{22}$ to elapse between the start of a deforming force and the rupture of an ACL in vitro.

More surprising than the improvements in the muscle reaction time was the significant slowing of the voluntary muscle reaction times in the medial hamstring and medial quadriceps muscles seen with isokinetic training. This apparent slowing is cause for concern. If this is accurate and if this finding is consistent in training studies performed at higher speeds, the adage that weight training negatively affects quickness and fine motor skills may be true. In the past, some coaches had feared weight training for athletes in finesse sports because they observed slowed or poorly coordinated activity performance with this type of training. For years, weight training was discouraged in basketball players and baseball pitchers where fine motor skills were emphasized, but it was encouraged in football linemen where power and strength were thought to be most important. At this time, however, our findings need to be kept in perspective.
Improvements in the time needed to reach peak muscle torque were seen with isokinetic training at $60 \mathrm{deg} / \mathrm{sec}$ in the hamstring, quadriceps, and gastrocnemius muscles. Muscle reaction time is only one parameter in a complex system that is not fully understood. Weight training obviously has many potential benefits, such as the improvements in time to peak muscle torque, and should not be discarded based on our findings alone.

The slower voluntary muscle reaction time seen after 6 weeks of isokinetic training in this study appears to contrast the work of Pipes and Wilmore, ${ }^{18}$ which suggests that isokinetic training improves motor task performance. Typical motor tasks include the vertical jump, the 40-yard dash, and the softball throw. In the study by Pipes and Wilmore, isokinetic training at $136 \mathrm{deg} / \mathrm{sec}$ produced more favorable results than either slower speed isokinetic training $(24 \mathrm{deg} / \mathrm{sec})$ or isotonic training, which produced no motor task improvements. Our report emphasizes the need to examine not only the mode but the speed of training when designing programs for sport-specific skills.

The apparent improvement in muscle reaction time and time to peak muscle torque seen with agility training may be attributed to the repetition of quick quadriceps-hamstring-gastrocnemius muscle contractions, which are needed to stabilize the knee joint during jumping, twisting, and pivoting. Although the isotonic and isokinetic programs did isolate and activate the same muscle groups, the emphasis on dynamic, coordinated muscle function was not the same. The isotonic and isokinetic programs used in this study emphasized strength acquisition and were performed at slower speeds than the agility training.

When tested isokinetically for peak torque, only knee extension and ankle plantar flexion in the isokinetic group improved significantly during the 6-week training period. The lack of improvement in knee flexion strength in this group was unexpected. However, the lack of isokinetic change in the agility and isotonic groups probably reflects the specificity of these training and testing modes. Although isotonic and agility performance improved during training, this improvement was not reflected in the results of the isokinetic strength tests. This lack of crossover is consistent with the results reported by Pipes and Wilmore, ${ }^{18}$ who used isokinetic and isotonic strength training in adult men.

In general, the strength improvements experienced in a 6-week program can probably be attributed to improvements in neural activation rather than muscle hypertrophy ${ }^{12,17,20}$ or improvements in body coordination. ${ }^{15,16,19}$ This neural explanation for torque improvements is also reflected in the time to peak muscle torque changes seen with training (Fig. 2).

The time to peak muscle torque data may be as important as the muscle reaction time data. To prevent injury, large muscle forces may be needed, making both muscle reaction time and time to peak muscle torque key parameters to study. At $60 \mathrm{deg} / \mathrm{sec}$, which is a slow speed compared with activities of daily living (e.g., walking is approximately 220 $\mathrm{deg} / \mathrm{sec}$ ), most muscle groups showed improvement in the time to peak muscle torque after 6 weeks of training. Interestingly, the control group improved by an average of 11 
msec, suggesting a learning effect with repeat testing. The agility group showed the largest change in the time to peak muscle torque for the quadriceps and hamstring muscles, although the changes were not significant (Fig. 2). Surprisingly, the isotonic group showed a slower hamstring muscle response after 6 weeks of training, which is difficult to explain. Häkkinen and Komi ${ }^{11}$ reported improvements in the time to peak muscle torque with isotonic training ( 3 times per week for 16 weeks) for the quadriceps muscles. This improvement was attributed to an increase in muscle spindle sensitivity.

Because the agility-trained group improved an average of $74 \mathrm{msec}$ in the time to peak muscle torque for the gastrocnemius muscle, this program may be best suited for training the gastrocnemius muscle. No other muscle in any training group showed such improvement at either isokinetic speed. Other specially designed programs may be able to challenge the quadriceps and hamstring muscles to a greater degree.

The lack of correlation seen between muscle reaction time and the time to peak muscle torque in this study is important to note and has been reported previously. ${ }^{14}$ Both parameters depend on neural pathways and occur sequentially. Both appear to be needed for effective limb protection but seem affected by different training factors.

Although it is tempting to draw conclusions regarding athletic training from this study, the muscle performance parameters measured are only two of the many indices available for evaluation and may not represent the most important factors in terms of knee joint stabilization and injury prevention. With these limitations in mind, it appears that agility training has the most beneficial effect on muscle reaction time, but agility and isokinetic training affect the time to peak muscle torque most significantly. Incorporation of such training techniques to prepare for sports that require jumping and pivoting appears justified at least until better indices of performance, other than muscle reaction time and time to peak muscle torque, can be identified.

In terms of knee laxity testing, with muscles relaxed and contracted, the minimal change in anterior tibial translation during 6 weeks of training was not surprising; no large change in laxity was expected. The isotonic group was the only group to show a statistically significant change $(0.83 \mathrm{~mm})$ in anterior tibial translation, and this change is probably not clinically significant.

One shortcoming of this study was that individuals were not randomly assigned to training and control groups. Individuals were allowed to select their training preferences and four participants (agility, one; isokinetic, three) dropped out during the 6 -week training period because of injuries.

\section{CONCLUSIONS}

Isokinetic, isotonic, and agility training regimens do appear to affect the muscle reaction time both positively and negatively in the quadriceps, hamstring, and gastrocnemius muscles in response to anterior tibial translation. Peak torque, time to peak torque, and endurance are independent variables affected by muscle training and may not correlate with muscle reaction time in performance evaluations. Agility training seems to produce the most desirable effects in muscle reaction time in this model of anterior tibial translation control, but agility and isokinetic training appear to improve the time to peak muscle torque the most consistently.

\section{REFERENCES}

1. Adeyanju K, Crews TR, Meadors WJ: Effects of two speeds of isokinetic training on muscular strength, power and endurance. J Sports Med Phys Fitness 23: 352-356, 1983

2. Barrack RL, Skinner HB: The sensory function of knee ligaments, in Daniel D, Akeson WH, O'Connor JJ (eds): Knee Ligaments: Structure, Injury, and Repair. New York, Raven Press, 1990, pp 95-114

3. Barrack RL, Skinner HB, Brunet ME, et al: Joint kinesthesia in the highly trained knee. J Sports Med Phys Fitness 24: 18-20, 1984

4. Barrack RL, Skinner HB, Cook SD: Proprioception of the knee joint. Am J Phys Med 63: 175-181, 1984

5. Beard DJ, Dodd CA, Trundle HR, et al: Proprioception enhancement for anterior cruciate ligament deficiency. J Bone Joint Surg 76B: 654-659, 1994

6. Coyle EF, Feiring DL, Rotkis TC, et al: Specificity of power improvements through slow and fast isokinetic training. J App/ Physiol 51: 1437-1442, 1981

7. Crockett JL, Edgerton VR, Max SR, et al: The neuromuscular junction in response to endurance training. Exp Neurol 51: 207-215, 1976

8. Curl WW, Markey KL, Mitchell WA: Agility training following anterior cruciate ligament reconstruction. Clin Orthop 172: 133-136, 1983

9. Francis PR, Tiptron CM: Influence of a weight training program on quadriceps reflex time. Med Sci Sports 1: 91-94, 1969

10. Gettman LR, Culter LA, Strathman TA: Physiologic changes after 20 weeks of isotonic vs isokinetic circuit training. $J$ Sports Med Phys Fitness 20: $265-274,1980$

11. Häkkinen K, Komi PV: Changes in neuromuscular performance in voluntary and reflex contraction during strength training in man. Int $J$ Sports Med 4: 282-288, 1983

12. Häkkinen $\mathrm{K}, \mathrm{Komi} P V$ : Electromyographic changes during strength training and detraining. Med Sci Sports Exerc 15: 455-460, 1983

13. Häkkinen $K$, Pakarinen $A$, Alen $M$, et al: Neuromuscular and hormonal adaptations in athletes to strength training in two years. $J$ App/ Physiol 16: 2406-2412, 1988

14. Ihara $H$, Nakayama $A$ : Dynamic joint control training for knee ligament injuries. Am J Sports Med 14: 309-315, 1986

15. Kottke FJ: From reflex to skill: The training of coordination. Arch Phys Med Rehabil 61: 551-561, 1980

16. Kottke FJ, Halpern D, Easton JKM, et al: The training of coordination. Arch Phys Med Rehabil 59: 567-572, 1978

17. Moritani T, DeVries HA: Neural factors versus hypertrophy in the time course of muscle strength gain. Am J Phys Med 58: 115-130, 1979

18. Pipes TV, Wilmore $\mathrm{JH}$ : Isokinetic vs, isotonic strength training in adult men. Med Sci Sports 7: 262-274, 1975

19. Rutherford OM, Jones DA: The role of learning and coordination in strength training. Eur J Appl Physiol 55: 100-105, 1986

20. Sale DG: Neural adaptation to resistance training. Med Sci Sports Exerc 20: S135-SI45, 1988

21. Wojtys EM, Huston LJ: Neuromuscular performance in normal and anterior cruciate ligament-deficient lower extremities. Am J Sports Med 22: 89-104, 1994

22. Yasuda $K$, Erickson AR, Johnson RJ, et al: Dynamic strain behavior in the medial collateral and anterior cruciate ligaments during lateral impact loading. Trans Orthop Res Soc 17: 127, 1992 